\title{
Workshop on Radio Transients
}

\author{
Organized by Steve Croft $^{1}$ \& Bryan Gaensler ${ }^{2}$ \\ ${ }^{1}$ Department of Astronomy, University of California, Berkeley, CA 94720, USA) \\ email: scroft@astro.berkeley.edu \\ ${ }^{2}$ Sydney Institute for Astronomy, The University of Sydney, NSW 2006, Australia
}

Summary. We are entering a new era in the study of variable and transient radio sources. This workshop discussed the instruments and the strategies employed to study those sources, how they are identified and classified, how results from different surveys can be compared, and how radio observations tie in with those at other wavelengths. The emphasis was on learning what common ground there is between the plethora of on-going projects, how methods and code can be shared, and how best practices regarding survey strategy could be adopted.

The workshop featured the four topics below. Each topic commenced with a fairly brief introductory talk, which then developed into discussion. By way of preparation, participants had been invited to upload and discuss one slide per topic to a wiki ahead of the workshop.

1. Telescopes, instrumentation and survey strategy. New radio facilities and on-going projects (including upgrades) are both studying the variability of the radio sky, and searching for transients. The discussion first centred on the status of those facilities, and on projects with a time-domain focus, both ongoing and planned, before turning to factors driving choices of instrumentation, such as phased array versus single pixel feeds, the field of view, spatial and time resolution, frequency and bandwidth, depth, area, and cadence of the surveys.

2. Detection, pipelines, and classification. The workshop debated (a) the factors that influence decisions to study variability in the $(\mathrm{u}, \mathrm{v})$ plane, in images, or in catalogues, (b) whether, and how much, pipeline code could potentially be shared between one project and another, and which software packages are best for different approaches, (c) how data are stored and later accessed, and (d) how transients and variables are defined and classified.

3. Statistics, interpretation, and synthesis. It then discussed how (i) the choice of facility and strategy and (ii) detection and classification schemes influence what is seen (in terms of types of object and rates) by different surveys, (iii) how results from different surveys could be compared, and (iv) how what we know from existing surveys drives choices (i) and (ii), particularly as regards finding new classes of object.

4. Multiwavelength approaches. The workshop concluded by discussing what information is needed from wavelengths other than radio in order to classify transients and variables adequately and predict their rates as a function of topics (1), (2) and (3). It asked what the constraints are on responding to, and issuing triggers for, follow-up observations, and how that might feed back into considerations for designing our telescopes and surveys. 